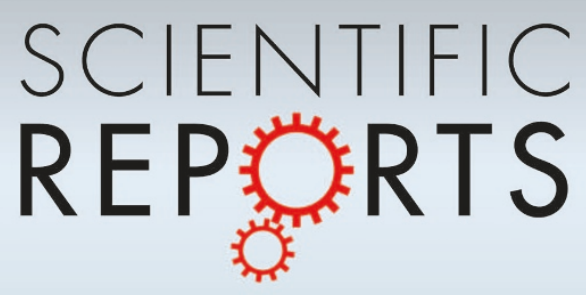

OPEN

SUBJECT AREAS:

GLASSES

METALS AND ALLOYS

MECHANICAL PROPERTIES

Received

10 February 2014

Accepted

2 April 2014

Published

23 April 2014

Correspondence and requests for materials should be addressed to

Q.W. lqingwang@

shu.edu.cn) or J.L.

(jianlu@cityu.edu.hk)

\section{Superior Tensile Ductility in Bulk Metallic Glass with Gradient Amorphous} \section{Structure}

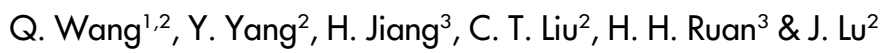

\begin{abstract}
'Laboratory for Microstructures, Institute of Materials Science, Shanghai University, Shanghai 200072, China, ${ }^{2}$ Centre for Advanced Structural Materials, Department of Mechanical and Biomedical Engineering, City University of Hong Kong, Tat Chee Avenue, Hong Kong, ${ }^{3}$ Department of Mechanical Engineering, The Hong Kong Polytechnic University, Hong Kong.
\end{abstract}

Over centuries, structural glasses have been deemed as a strong yet inherently 'brittle' material due to their lack of tensile ductility. However, here we report bulk metallic glasses exhibiting both a high strength of $\sim 2 \mathrm{GPa}$ and an unprecedented tensile elongation of $2-4 \%$ at room temperature. Our experiments have demonstrated that intense structural evolution can be triggered in theses glasses by the carefully controlled surface mechanical attrition treatment, leading to the formation of gradient amorphous microstructures across the sample thickness. As a result, the engineered amorphous microstructures effectively promote multiple shear banding while delay cavitation in the bulk metallic glass, thus resulting in superior tensile ductility. The outcome of our research uncovers an unusual work-hardening mechanism in monolithic bulk metallic glasses and demonstrates a promising yet low-cost strategy suitable for producing large-sized, ultra-strong and stretchable structural glasses.

G lasses have been existent in our society for thousands of years. They came along from the ancient time to our modern world as a very important class of engineering materials ${ }^{1}$. Some of them could be readily seen in our daily life, such as window pane (ceramic glass) and unfilled epoxy resin (polymeric glass); and some of them already became a necessity or showed a great potential for today's nano- and bio-technology, such as amorphous silicon and amorphous metals, also known as metallic glass ${ }^{2,3}$. Despite the technological importance of these structural glasses, they have been suffering a similar kind of problem since their advent. Strong glasses, such as ceramic and metallic glasses, are not stretchable in tension, usually failing catastrophically upon yielding; whilst soft glasses, such as polymeric glasses, are stretchable but their strength is just too low to be considered in most structural applications. For materials scientists, this strength-ductility paradox is a longlasting problem, which is yet to be tackled for structural glasses despite that substantial efforts have been paid over the past decades ${ }^{4-7}$.

Making a strong yet stretchable glass poses a big challenge to materials scientists. Unlike crystals whose properties are tunable via microstructural engineering, glasses inherit an amorphous structure from their liquid melts. Due to the lack of crystalline-like defects, like dislocations, glasses are generally much stronger than their crystalline counterparts; however, they are also plagued by the structural amorphousness and usually display no ductility in a uniaxial mechanical test ${ }^{8,9}$. This brittle fracture mode has prompted people to seek solutions by combining monolithic glasses with secondary 'phases', such as crystalline inclusions or voids/pores, in order to attain ductility through the interaction of these secondary 'phases' with the otherwise catastrophically growing defects, such as shear bands or cracks ${ }^{4-6,10,11}$. Although ductility enhancement could be hence achieved, however, this 'composite' approach is strength-sacrificing or usually destroys the overall structural amorphousness ${ }^{5,11}$, which therefore limits its use in the applications where a fully amorphous structure is demanded, such as the recently emerging metallic-glass based bio-technology ${ }^{3,12}$. In this Article, we would like to demonstrate that, by using a low-cost and carefully controlled surface processing technique, we could render strong glasses with superior tensile ductility without strength degradations at the macroscopic scale.

The model glass we used is the bulk metallic glass (BMG) with the chemical composition of $\mathrm{Cu}_{46} \mathrm{Zr}_{47} \mathrm{Al}_{7}$ (in atomic \%), which was obtained in a plate form using the Cu-mold suction casting method (see Method). Following sample preparation, the Surface Mechanical Attrition Treatment (SMAT) was performed on the BMG plates using an in-house developed ultrasonic system (see Method). Afterwards, various means for structural characterization were applied to examine the BMG structure before and after the SMAT process (see 
Method). For mechanical characterization, dog-bone shaped tensile specimens, with the gauge dimensions of $3 \mathrm{~mm} \times 1 \mathrm{~mm} \times 0.4 \mathrm{~mm}$, were carved out of the as-cast and SMATed BMG plates. Tensile tests were then performed at room temperature and at a constant strain rate of $10^{-4} \mathrm{~s}^{-1}$. To avoid experimental artifacts resulting from sample slippage in fixtures, tensile strain was measured by the digital image correlation (DIC) method (see Method). After tensile fracture, the surface morphologies of all specimens were investigated by electron microscopy.

\section{Results}

Surface Mechanical Attrition Treatment (SMAT). The SMAT process was performed using an in-house developed ultrasonic system with 512 2-mm stainless steel balls, in which the balls were excited by an ultrasonic horn of $20 \mathrm{KHz}$ frequency [Fig. 1(a)]. The velocities of the balls were estimated to be on the order of $10 \mathrm{~m} / \mathrm{s}$, which can result in a strain rate up to $\sim 10^{3} \mathrm{~s}^{-1}$ in the treated BMGs $\mathrm{s}^{13}$. After SMAT, the treated surface of the BMGs became relatively rougher with a root-mean-square (RMS) roughness of $\sim 360 \mathrm{~nm}$, but still looks much smoother than that after the classic shot peening process ${ }^{14}$, as shown in Fig. 1(b). Through the bondedinterface technique ${ }^{15}$, the formation of profusive subsurface shear bands were observed corresponding to the different treatment times, $\Delta t$. As shown in Figs. 1(c) to (f), it can be seen that the size of the SMAT affected zone increases with $\Delta t$ but seemingly saturates at $\sim 210 \mu \mathrm{m}$ when $\Delta t>40$ minutes [Fig. 1(g)]. Furthermore, extensive X-ray diffraction tests were carried out on the surfaces of the different BMG samples. As seen in Fig. 1(h), the broad peaks of the XRD curves indicate that the overall structural amorphousness was retained even after the 60-minute SMAT. As such, we can rule out any possibilities that secondary crystalline phases might have formed in the SMATed BMGs.

Mechanical Characterization of As-Cast versus SMATed BMG. Figure 2(a) displays the true stress-strain curves obtained from the as-cast and SMATed BMG samples using the DIC method (See Supplementary Information). From these curves, one can clearly (a)

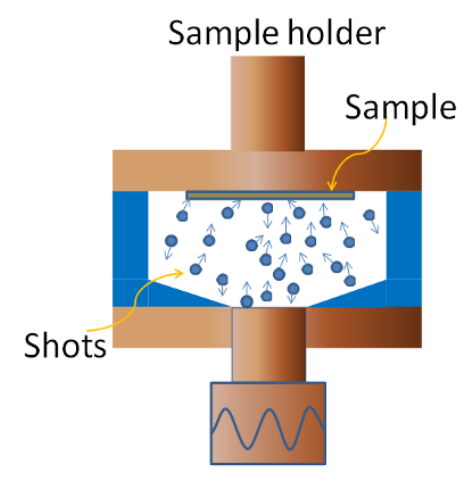

Ultrasonic vibration generator (b)
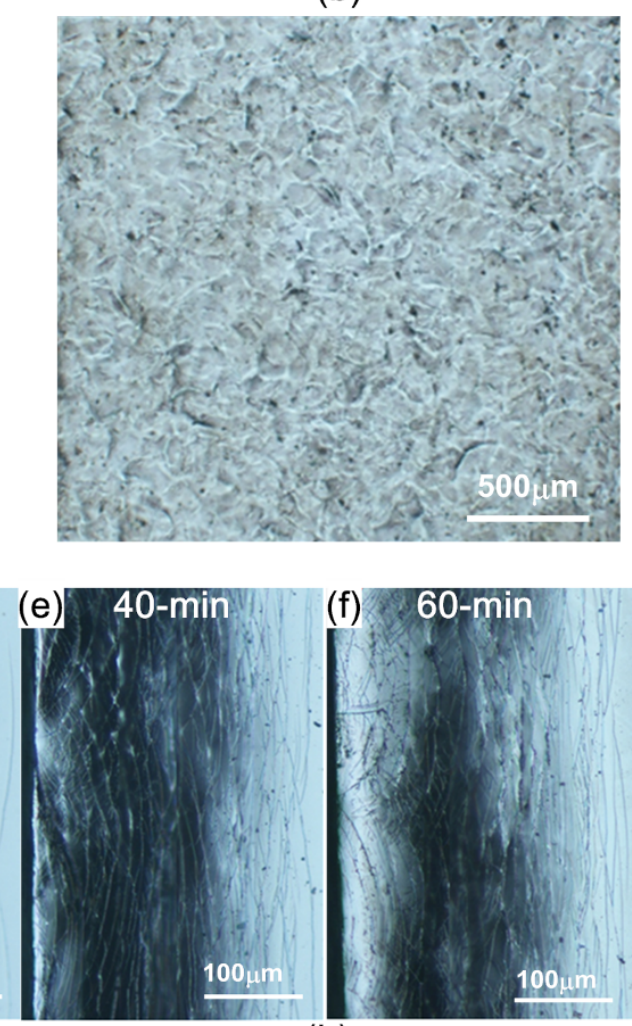

(h)

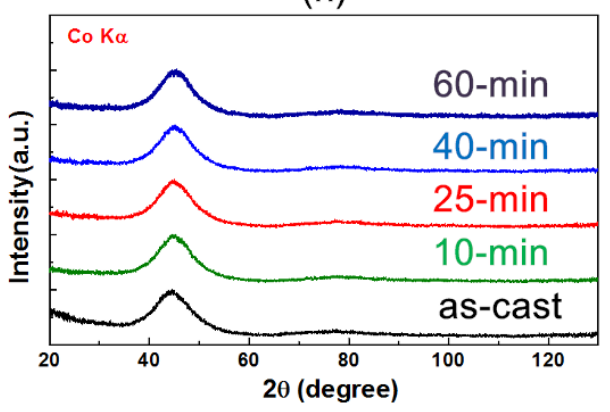

Figure $1 \mid$ The experimental data related directly to the SMAT process. (a) The schematics of the experimental set-up of SMAT, (b) the optical microscopy (OM) image of the surface of a $0.4 \mathrm{~mm}$ thick $\mathrm{Cu}-\mathrm{Zr}$-Al BMG plate after 60-min SMAT exhibiting a large number of visible craters, the OM images obtained from the bonded interface technique showing the subsurface profusive shear banding in the BMG samples after SMAT for (c) 10, (d) 25, (e) 40 and (f) 60 minutes, (g) the variation of the SMAT-affected zone size with the treatment time $\Delta t$ as inferred from the OM images, and (h) the X-ray diffraction (XRD) curves confirming the amorphous structure of all BMG samples before and after the SMAT processes. 
(a)
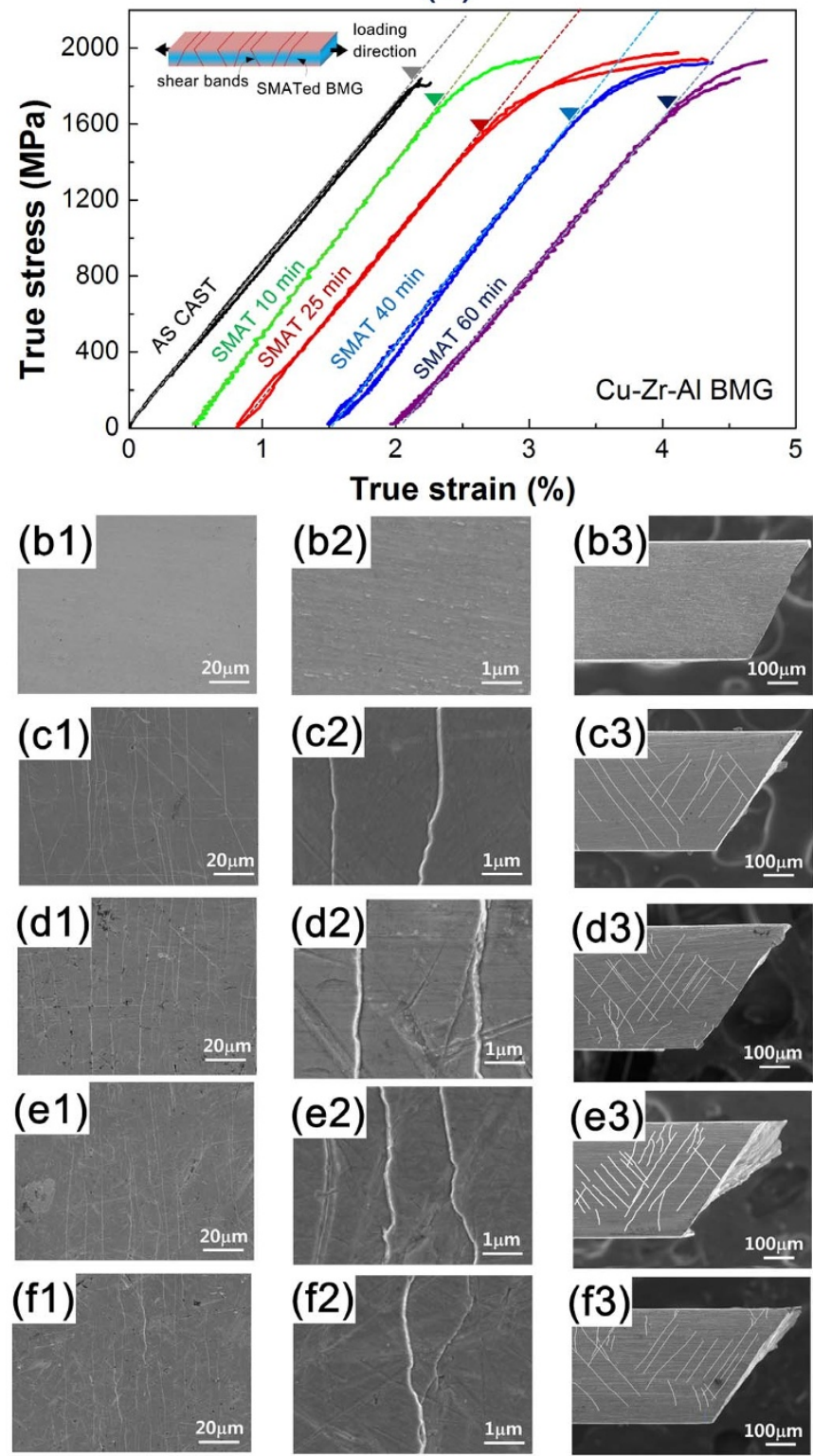

Figure $2 \mid$ Mechanical characterization of the as-cast and SMATed 0.4$\mathrm{mm}$ thick $\mathrm{Cu}_{46} \mathrm{Zr}_{47} \mathrm{Al}_{7}$ metallic glass. (a) The room-temperature true stress-strain curves obtained at the strain rate of $1 \times 10^{-4} \mathrm{~s}^{-1}$ (the BMG yield strengths are marked at the departure of the stress-curve curve from a linear response), (bi) - ( $\mathrm{f} i$ ) the scanning electron microscopy (SEM) images of the as-cast and SMATed BMG tensile samples after fracture $(i=1,2,3$ in which $1=$ the top view, $2=$ the enlarged top view and $3=$ the cross sectional view where the shear traces are marked), and (g) the schematics of the effective shear-banding model that was used to back calculate the total tensile ductility of the SMATed BMG.

see that the as-cast samples do not possess any tensile ductility and fracture instantly upon yielding, which is similar to what have been reported in the literature ${ }^{9}$; in sharp contrast, the SMATed samples exhibit a varying degree of unusual strain hardening and hence tensile ductility before the final failure. Impressively, the tensile ductility of $\sim 2 \%$ was obtained after the sample was treated by 25 minutes. In contrast, the measured tensile ductility decreased when the BMG was under- or over-treated. Furthermore, it is noted that the yield strength of the SMATed BMG decreases somewhat but the fracture strength significantly increases with the rising tensile ductility. This unique feature of deformation provides importance evidence to understand the physical mechanisms underlying the ductility enhancement in the SMATed BMGs, as will be discussed in the later text.

Despite the different tensile ductility measured, however, all BMG samples ultimately fractured via the same mode, viz. cracking along an inclined plane as shown by the scanning electron microscopy (SEM) images [Figs. 2(b3) to (f3)]. Post-mortem imaging analyses showed that nearly no shear band is visible on the surface of the ascast samples [Figs. 2(b1)-(b3)]; by comparison, the outer surfaces of the SMATed samples were populated with a large number of shear bands, as shown in Figs. 2(c1) - (f3). These SEM observations suggest that the tensile ductility in the SMATed BMG probably results from multiple shear banding. If that was the case, we may back calculate the tensile ductility by invoking an effective shear offset model ${ }^{16}$, as shown by the inset of Fig. 2(a), which simply assumes that the tensile plastic strain $\varepsilon_{p}$ is accommodated through the shear-band displacements, or shear offsets, that were formed on the sample surface. In that regard, we can write:

$$
\varepsilon_{p}=\sum_{i=1}^{N}\left(\lambda_{i} \cos \theta / L_{0}\right)=\rho \bar{\lambda} \cos \theta
$$

where $N$ is the total number of visible shear bands, $\lambda_{i}$ the shear offset of the $i^{t h}$ shear band, $L_{0}$ the sample gauge length, $\theta$ the angle between the shear plane and the loading direction, $\rho$ the density of the visible shear bands, and $\bar{\lambda}$ the average shear offset. Table 1 lists the calculated tensile ductility of the SMATed BMG in comparison with the measured values, from which it can inferred that, despite the limited resolution of the SEM images and the simplicity of the effective shear-offset model ${ }^{16}$, the calculated $\varepsilon_{p}$ generally agrees with the measured $\varepsilon_{p}$. This agreement thus confirms that multiple shear banding is the main source of the tensile ductility as witnessed in the tensile tests.

Residual Stress Distribution and Dynamic Structural Evolution. Like shot peening, the SMAT process gave rise to residual stresses in the treated BMG. By using the classic hold-drilling method ${ }^{17-20}$, the residual stress distribution along the sample thickness direction was measured for the as-cast and SMATed BMGs (see Method). Interestingly, it was found that the most ductile BMG after the 25minute SMAT exhibits a residual stress distribution drastically different from the others, which peaks at a compressive stress of $\sim 500 \mathrm{MPa}$ at the $30-\mu \mathrm{m}$ distance from the impacted surface and then transits quickly to the tensile residual stress, as shown in Fig. 3(a). By comparison, the other residual stress profiles either are too 'flat', with the maximum compressive residual stress ranging from -50 to $-100 \mathrm{MPa}$, or possess a spread-out peak located much deeper than the 25-minute one. As compared to the residual stress profiles usually measured in the shot-peened crystalline metals, which saturates with the dislocation density and thus the treatment time, the conspicuous variation in the residual stress profiles, as measured from the SMATed BMGs, is unusual, which may signal an intense structural evolution process being triggered by the SMAT process.

In principle, severe plastic deformation, as expected for the SMAT process, could result in rejuvenation or further 'disordering' of the BMG atomic structure ${ }^{21}$, which is accompanied by volume dilation. As this occurs, the outer layer of the treated BMG expands relative to the inner one, therefore giving rise to a compressive residual stress near the surface while a tensile residual stress inside. Here, we envision that structural relaxation may also take place with the prolonged treatment time, such as $\Delta t>25$ minutes, which counteracts structural rejuvenation, thus homogenizing the 'disorderness' of the amorphous structure and smoothing out the residual stress profile, as shown in Fig. 3(a). 
Table 1 | Comparison of the tensile ductility $\varepsilon_{p}$ measured from the stress-strain curve and that calculated based on the measured average shear-band spacing $\Delta s$, shear-band density $\rho$, shear-band offset $\bar{\lambda}$, and shear angle $\theta$

\begin{tabular}{|c|c|c|c|c|c|c|c|}
\hline & & & & & & $\varepsilon_{p}(\%)$ & $\varepsilon_{p}(\%)$ \\
\hline & $\Delta s(\mu \mathrm{m})$ & $\rho=1 / \Delta \mathrm{s}(1 / \mu \mathrm{m})$ & $\bar{\lambda}(\mu \mathrm{m})$ & $L_{0}(\mu \mathrm{m})$ & $\theta$ (deg) & Calcu & Exp \\
\hline As-cast & 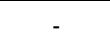 & - & - & 3000 & $55-60$ & 0 & 0 \\
\hline $10 \mathrm{~min}$ & $34 \pm 5$ & $0.029 \pm 0.004$ & 0.2 & 3000 & 55 & $0.34 \pm 0.05$ & 0.81 \\
\hline $25 \mathrm{~min}$ & $11 \pm 2$ & $0.091 \pm 0.008$ & 0.2 & 3000 & $52-55$ & $1.07 \pm 0.1$ & $1.5 \pm 0.3$ \\
\hline $40 \mathrm{~min}$ & $16 \pm 2$ & $0.061 \pm 0.008$ & 0.2 & 3000 & $54-56$ & $0.68 \pm 0.05$ & $0.89 \pm 0.04$ \\
\hline $60 \mathrm{~min}$ & $28 \pm 3$ & $0.036 \pm 0.004$ & 0.2 & 3000 & $53-55$ & $0.41 \pm 0.08$ & $0.79 \pm 0.05$ \\
\hline
\end{tabular}

To verify the above idea, differential scanning calorimetry (DSC) experiments were carried out. Figure 3(b) shows a set of DSC curves obtained from the as-cast and SMATed BMGs. A distinct exothermic peak can be observed on each of these curves, indicating that a remnant excess enthalpy was released during continuously heating at the constant rate of $20 \mathrm{~K} / \mathrm{min}$. This enthalpy recovery is followed by a distinct endotherm at a temperature close to the glass transition point $T_{g}$, and then a crystallization exotherm at a higher temperature of $T_{x}$, which is similar to what could be observed in regular glasses ${ }^{22}$. From these DSC curves, important thermodynamic properties, such as relaxation and crystallization enthalpy, could be derived, from which one can infer the SMAT induced structural evolution process in the treated BMGs. As shown in Fig. 3 (c), the values of crystallization enthalpy for the SMATed samples are nearly identical to that of the as-cast, which again confirms the overall structural amorphousness of the SMATed samples. However, before crystallization occurs, it could be inferred that the amorphous structure of the BMG has been already altered by SMAT. As shown in Fig. 3(d), the value of relaxation enthalpy $\Delta H_{\text {relax }}$ significantly increases with the SMAT duration, rising rapidly from $-3.54 \mathrm{~J} / \mathrm{g}$ for the as-cast sample to $-5.86 \mathrm{~J} / \mathrm{g}$ for the one subjected to the $25-\mathrm{min}$ treatment. According to Ref. 22, this enthalpy releases indicate a free-volume raising process or a rejuvenated amorphous structure after SMAT. However, the value of $\Delta H_{\text {relax }}$ is reduced and tends to saturate at (a)

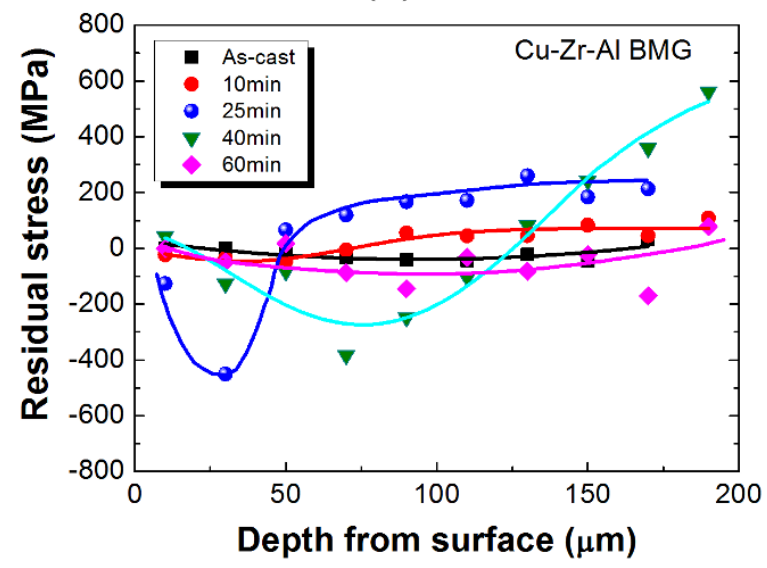

(c)

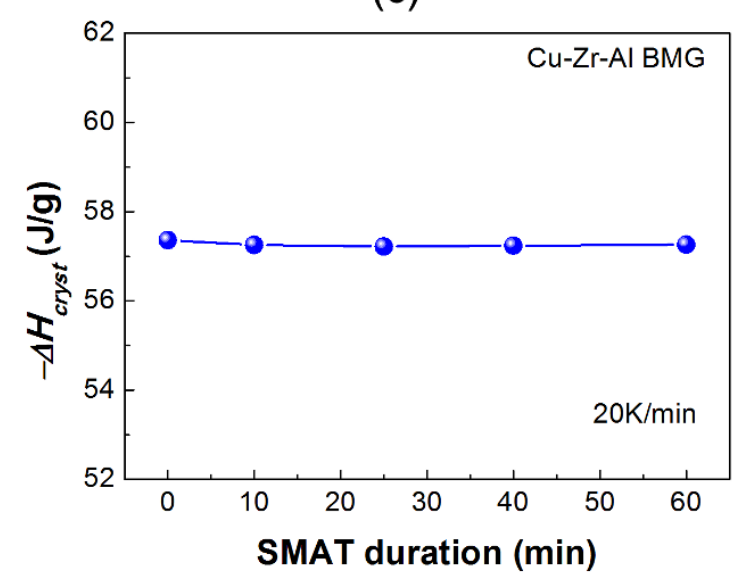

(b)

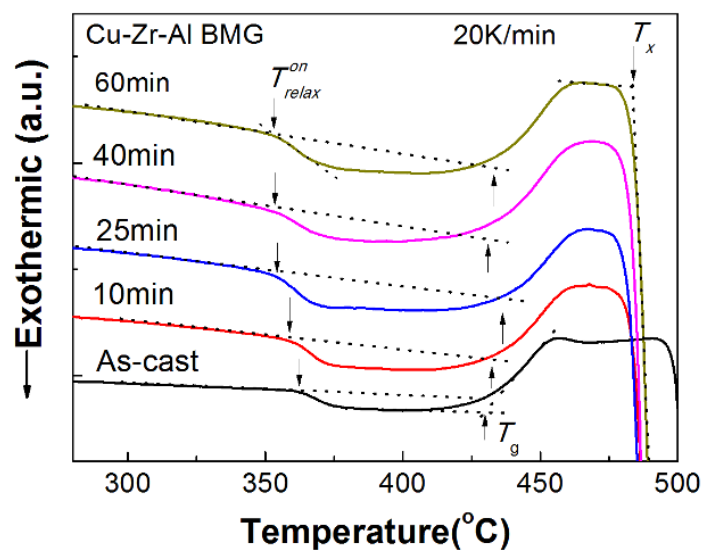

(d)

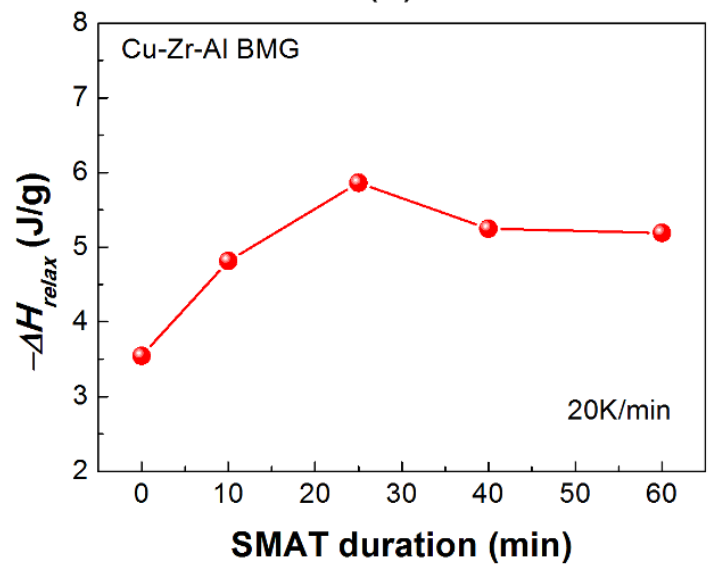

Figure $3 \mid$ The combined structural and mechanical effects of SMAT on the BMG. (a) The variation of the residual stress distribution along the sample thickness with the SMAT time, (b) the DSC curves obtained from the as-cast and SMATed BMGs, (c) the variations of the crystallization temperature $T_{x}$ and crystallization enthalpy $\Delta H_{\text {cryst }}$ with the treatment time, and (d) the variations of the onset relaxation temperature $T^{o n}{ }_{r e l a x}$ and relaxation enthalpy $\Delta H_{\text {rex }}$ with the treatment time. 
about $-5.2 \mathrm{~J} / \mathrm{g}$ as the treatment time is further prolonged. This behavior indicates the operation of a structural relaxation process, as previously envisioned.

Structural Characterization with Transmission Electron Microscopy. Next, extensive transmission electron microscopy (TEM) analyses were performed to characterize the amorphous structure at the different depths below the impacted surface of the SMATed BMG, as illustrated in Fig. 4(a). Specifically, Figures 4(b) - (d) display the TEM images obtained from the BMG after the 25-minute treatment. Very interestingly, one can clearly see a grain-like microstructure with a dark-bright contrast at a distance of $\sim 10 \mu \mathrm{m}$ below the impacted surface. The sizes of the dark regions range from 100 to $200 \mathrm{~nm}$ while those of the bright ones from 10 to $50 \mathrm{~nm}$, as seen in Fig. 4(b). At a deeper distance of $\sim 30 \mu \mathrm{m}$, the sizes of the dark regions are enlarged to $2-10 \mu \mathrm{m}$ while those of the bright ones, however, remain almost unaltered [Fig. 4(c)]. The gradient microstructural features ultimately vanish at the distance of $\sim 120 \mu \mathrm{m}$ [Fig. 2(d)]. According to the corresponding selective area electron diffraction (SAED) patterns [the insets of Figs. 4(b) - (d)] and also the high-resolution TEM [Fig. 2(g)], we confirm that the observed microstructures are all structurally amorphous.

The emergence of such a gradient amorphous structure can be rationalized by the theory of Bei et al..$^{23}$, who proposed that shear-band networking may produce an amorphous grain-like microstructure in BMGs, viz., the sheared zones tend to form the boundary-like regions through percolation, which possess a relatively low density due to the increased disorder thereof, and the un-sheared regions thus appear as the dark regions with a relatively high density. Considering the gradient shear-band density as exposed on the cross section of the SMATed BMGs [Fig.1(c) - (f)], the hypothesis of Bei et $\mathrm{al}^{23}$. provides a reasonable explanation for the gradient amorphous microstructures seen in Figs. 4(b) - (e).

To further testify the above hypothesis, nanoindentation tests were carried out across the sample thickness. As shown in Fig. 4(f), a BMG sample with only one surface treated was used for the hardness measurement. To avoid the influence of materials pile-up, the hardness was measured through directly imaging the indent size by following the method detailed in Ref. 24. Figure 4(g) shows the hardness so obtained as a function of the distance to the impacted surface. In accord with the structural features seen in the TEM images, the hardness reduces as the indentation approached the impacted surface. Considering the presence of a compressive residual stress near the impacted surface, which can boost up the apparent hardness ${ }^{25}$, we expect an even greater reduction in the intrinsic strength of the SMATed BMG, should the residual stress effect be removed. Therefore, this finding supports the hypothesis that the amorphous microstructure was formed due to profusive shear banding.

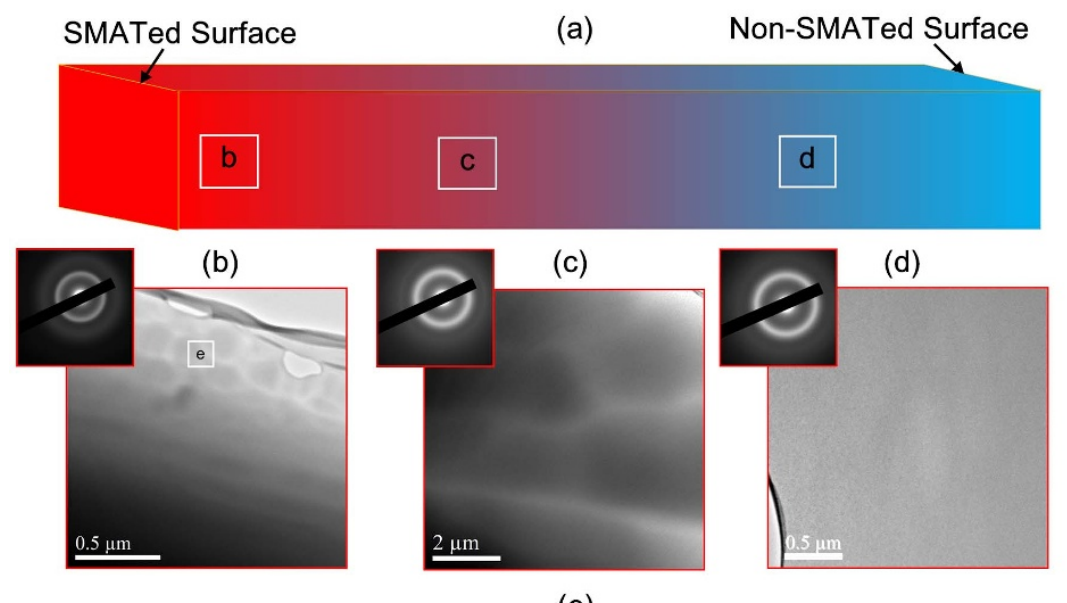

(e)
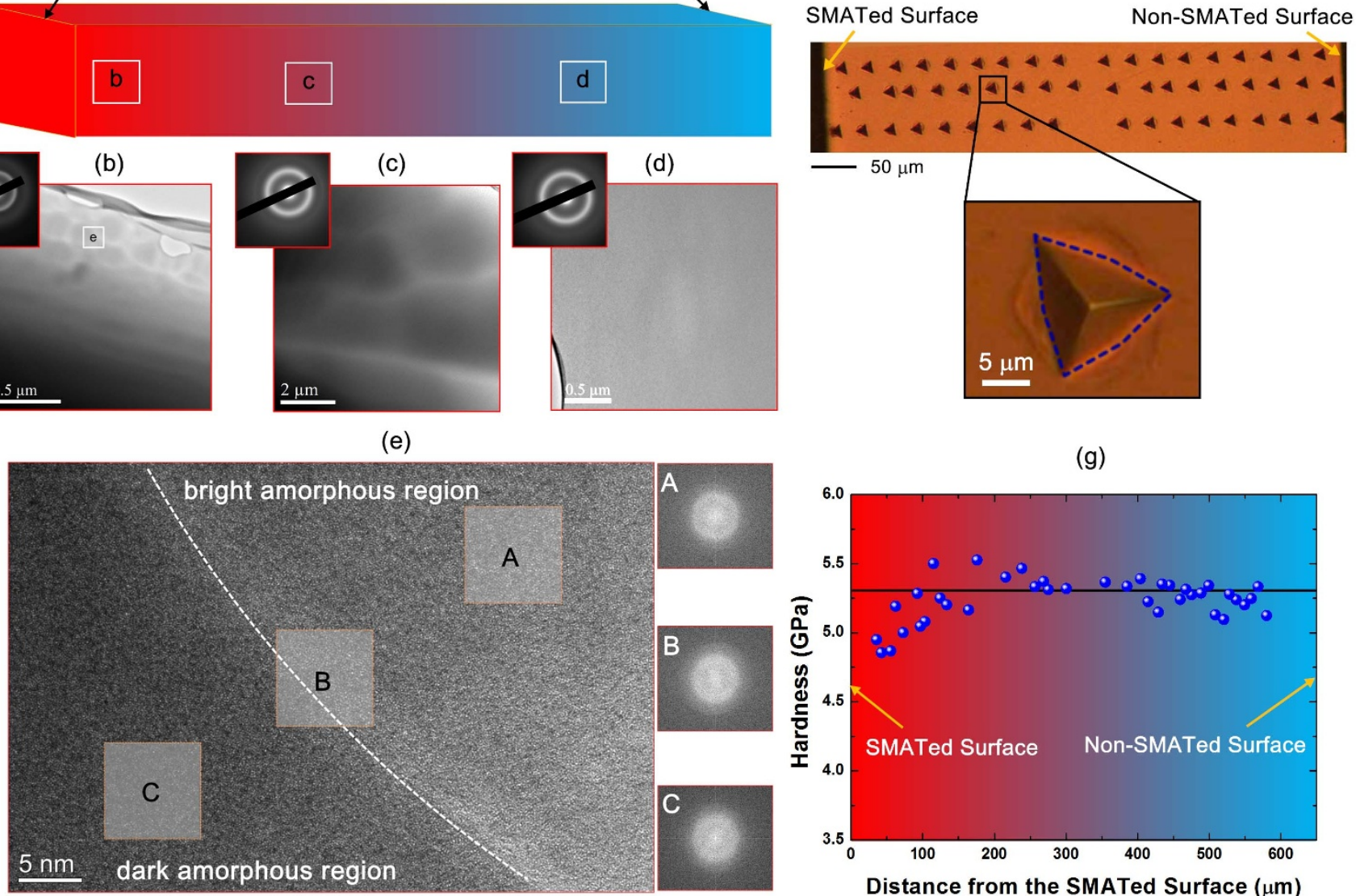

(g)

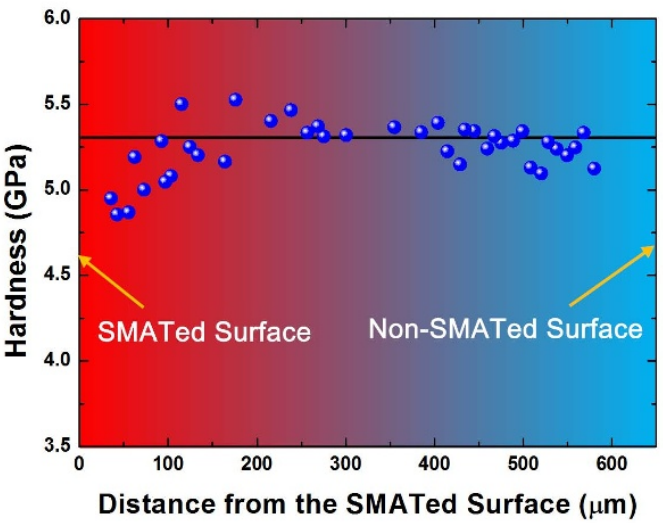

Figure $4 \mid$ The gradient amorphous structure in the 25-minute treated BMG. (a) the schematics showing the SMATed BMG undergoing a varying degree of structural evolution at the different distances to the impacted surface, (b) - (d) the TEM images of the atomic structure at the different depths [inset $=$ the selective area electron diffraction (SAED) pattern], and (e) the HRTEM image of the amorphous structure shown in (b). The inclined dashed line indicates the boundary between the dark amorphous region and the bright amorphous boundary-like region. The FFT patterns taken from the three random sites, labeled as $\mathbf{A}, \mathbf{B}$, and $\mathbf{C}$, confirm that the atomic structures are all structurally amorphous. (f) the indentation marks left on the cross section of the SMATed BMG (inset: the indentation mark with significant pile up) and (g) the variation of the indentation hardness with the distance from the SMATed surface. 


\section{Discussion}

Recently, it was shown that one could obtain bendable and malleable macro-sized glasses through residual stress engineering ${ }^{14}$ or stretchable nano-sized glasses ${ }^{7,26}$ through size reduction. Despite all these successes, one last question still remains, i.e., can we make strong and stretchable glasses at the macroscopic scale? To materials scientists, this is not only a question of scientific curiosity but also a matter of great technologic importance. Today, many important industrial innovations demand large-sized glasses, such as display technology, energy harness and bio-technology ${ }^{1,12}$. From the mechanical viewpoint, a uniform tensile stress represents the worst case of scenario one may encounter in a normal structural application; therefore, it is necessary to address the above question before glasses could be used safely and widely.

With the extensive experimental efforts as discussed above, it is clear that the tensile ductility comes along with a gradient amorphous microstructure, which promotes multiple shear banding while suppresses shear cracking at room temperature. On a qualitative basis, this may be attributed to an already 'softened' BMG surface, which offers easy sites for shear band nucleation as suggested in the previous work ${ }^{23}$. However, the simple argument cannot explain why cold-rolled or pre-compressed BMGs display no tensile ductility at room temperature ${ }^{27,28}$. After all, the similar behavior of surface softening was detected after all these mechanical pre-treatments. In that regard, a deeper understanding is needed for rationalizing our current results. In what follows, we will provide an explanation by invoking a physical model ${ }^{29}$ recently developed based on the structural heterogeneity in metallic glasses ${ }^{30-33}$.

According to the previous work ${ }^{30-33}$, it is known that the amorphous structure of BMGs is heterogeneous, which consists of liquidand solid-like atoms as illustrated in Fig. 5(a). Upon mechanical loading, the liquid-like atoms behave like inelastic inclusions, giving birth to local plastic events also known as shear transformations ${ }^{34}$; while the solid-like atoms behave as a whole like an elastic 'matrix' upholding the structural integrity of the BMG. Based on this understanding, a micromechanical model was recently developed to account for the lack of tensile ductility in $\mathrm{BMGs}^{29}$. According to this model $^{29}$, there are two atomic-scale inelastic deformation mechanisms competing with each other to control the fracture mode in a tensile test, i.e. cavitation in the elastic 'matrix' results in cracking and brittle fracture [Fig. 5(b)] while coalescence of the liquid-like atoms results in shear banding and ductile deformation [Fig. 3(c)]. Accordingly, the material strength for cavitation and cracking $\sigma_{f}$, and that for shear banding $\sigma_{s}$, can be derived, respectively, $\mathrm{as}^{29}$ :

$$
\sigma_{f}=\sqrt{f_{L L A}} \sigma_{0}
$$

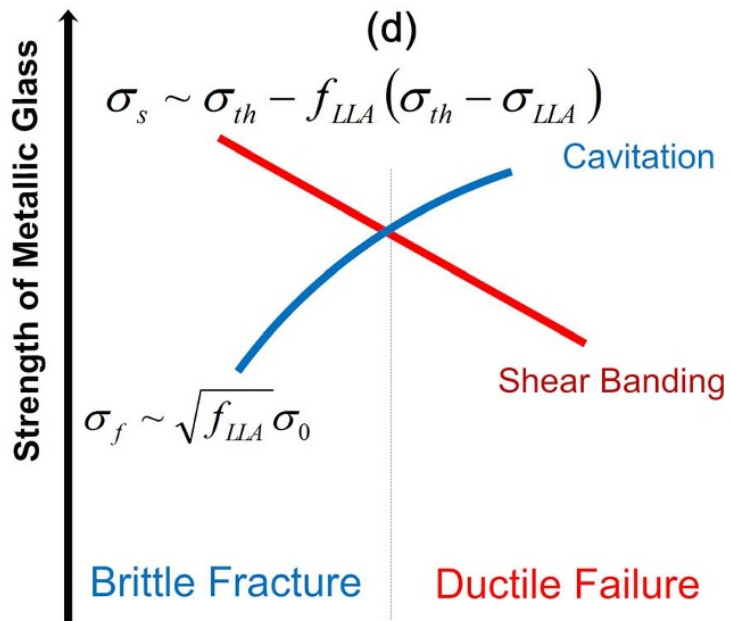

Volume Fraction of Liquid-like Regions

(e)

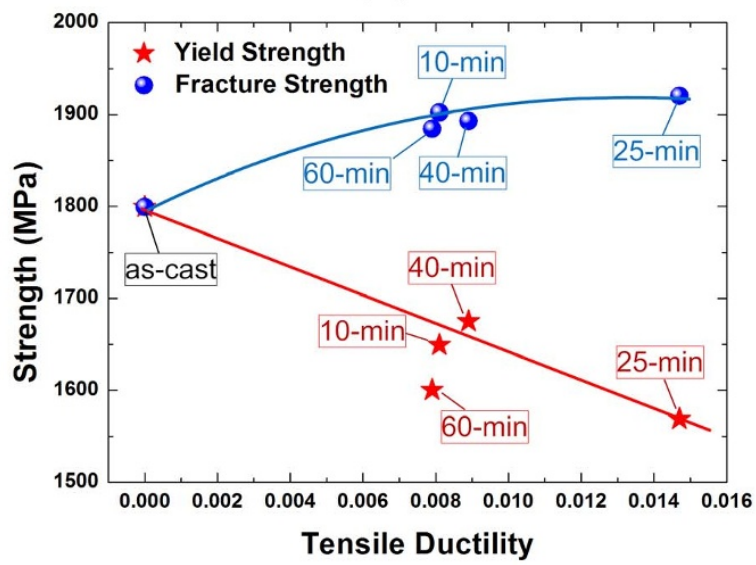

(a)

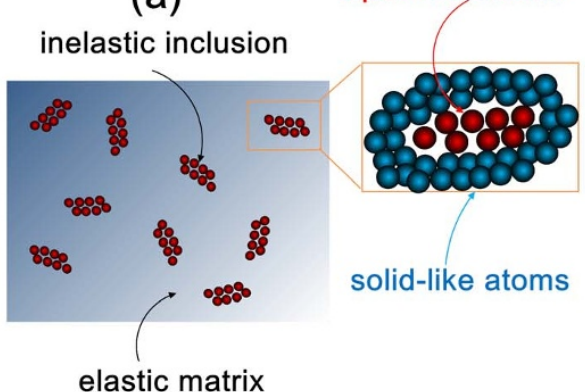

(b)

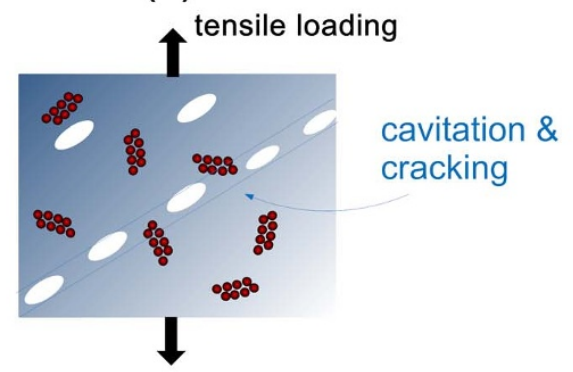

(c)

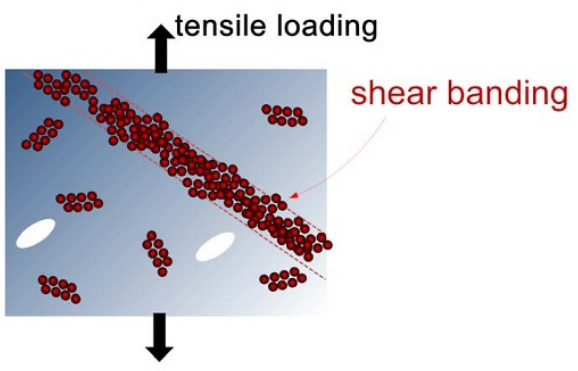

Figure 5 The proposed deformation mechanisms for the brittle to ductile transition in tension tests of bulk metallic glasses according to Ref. 29. (a) The schematics illustrating the structural heterogeneity in metallic glass consisting of liquid-and solid-like atoms, the sketched mechanisms of (b) the brittle fracture in BMG due to cavitation and cracking at a low volume fraction of liquid-like atoms, and (c) the ductile deformation in BMG due to shear banding at a high volume fraction of liquid-like atoms, and (e) the trend of the experimentally obtained yield and fracture strengths with the tensile ductility showing excellent agreement with the theory ${ }^{29}$. 


$$
\sigma_{s}=\sigma_{t h}-\left(\sigma_{t h}-\sigma_{L L A}\right) f_{L L A}
$$

where $f_{L L A}=$ the volume fraction of liquid-like atoms; $\sigma_{0}=$ the reference strength against cavitation in metallic glass; $\sigma_{t h}=$ the theoretical strength of the solid-like atoms; and $\sigma_{L L A}=$ the strength related to shearing of liquid-like atoms.

According to Eqs. (2) and (3), $\sigma_{f}$ and $\sigma_{s}$ vary with $f_{L L A}$ in an opposite manner. In such a case, brittle fracture corresponds to $\sigma_{f}$ $<\sigma_{y}$ for $f_{L L A} \sim 0$, and ductile deformation to $\sigma_{f}>\sigma_{y}$ for $f_{L L A} \sim 1$. In general, the brittle-to-ductile transition (BDT) takes place when $f_{L L A}$ reaches a critical value $f_{c}$. According to the previous work ${ }^{29}, f_{c} \sim 50 \%$ for the $\mathrm{Cu}-\mathrm{Zr}$ or Zr-based BMGs with $v \sim 0.36$. Nevertheless, $f_{L L A}$ is low in the as-cast BMGs, which is usually less than $20 \%$ according to the atomic-stress theory ${ }^{30,35,36}$. As such, brittle fracture seems inevitable in the tensile test of BMGs, as noticed since the $1970 \mathrm{~s}^{8,9,37}$.

However, in our SMATed BMGs, the amorphous structure is severely deformed near the surface. Under the condition of constrained plastic deformation, the liquid-like atoms now percolate giving rise to the gradient amorphous structures. As a result, the local $f_{L L A}$, as indicated by the fraction of the bright regions [Fig. 4(b) - (c)], can reach up to $50-70 \%$ near the surface, thus bypassing the BDT limit of $\sim 50 \%{ }^{29}$. Consequently, shear banding is preferred over cracking, which leads to the tensile ductility in the SMATed BMGs. Since the SMATed amorphous structure is not uniform, multiple shear banding develops until the potential shear banding sites with the high $f_{L L A}(>50 \%)$ are exhausted. When the flow stress reaches the fracture strength, shear banding again gives way to cracking and hence brittle fracture ensues. From a phenomenological perspective, this manifests as a strain hardening process as seen in Fig. 2(a). As shown in Fig. 5(e), the measured yield strength $\sigma_{y}$ indeed decreases while the corresponding fracture strength $\sigma_{f}$ increases with the tensile ductility. This trend finds excellent agreement with the theory.

Before closing, it is worth mentioning that the sharply peaked residual stress profile may help suppress cavitation, as discussed in the previous work ${ }^{14}$, and thus contributes to the enhancement of the tensile ductility. However, the SMATed BMGs after the 60-minute treatment exhibit limited but still appreciable tensile ductility compared to the as-cast despite that the measured two residual stress profiles are almost indistinguishable. Furthermore, it is known that compressive residual stress promotes the local yield strength or hardness; nevertheless, for the case of 25 min SMAT, the local yield strength decreases near the surface, which contradicts the residual stress effect but hints that structural rejuvenation overwhelms residual stress in altering the local plastic behavior of the SMATed BMG. From these regards, we believe that the role played by the residual stress must be supplementary to that of the microstructure change. Finally, we should emphasize that our current approach is applicable to other alloy compositions. Following the similar method, we also treated a Zr-based BMG with the chemical composition of $\mathrm{Zr}_{55} \mathrm{Cu}_{30} \mathrm{Al}_{10} \mathrm{Ni}_{5}$. Surprisingly, the SMATed Zr-based BMG exhibited a tensile ductility of nearly $\sim 4 \%$, almost twofold of what we already achieved in the Cu-Zr-based BMG (see Supplementary Information).

To conclude, we herein demonstrate that the brittle fracture in BMGs under tensile loading can be overcome by introducing a gradient amorphous structure through the carefully controlled SMAT process. Owing to the high volume fraction of the liquid-like atoms near the surface, shear banding is promoted while cavitation/fracture is suppressed, thus giving rise to the tensile ductility which cannot be obtained otherwise in the as-cast BMGs. Most importantly, the tensile ductility enhancement so obtained does not sacrifice but rather boosts the fracture strength of the glass, which somewhat reconciles the longstanding strength-ductility paradox facing BMGs. Since our method is in principle applicable to different alloy compositions, we envision that tensile ductility higher than $2-4 \%$ may be achievable in future, therefore paving the way for the structural applications of BMGs.

\section{Methods}

Material Fabrication. Alloy ingots with a nominal composition of $\mathrm{Cu}_{46} \mathrm{Zr}_{47} \mathrm{Al}_{7}$ were prepared by arc-melting mixtures of pure metals in Ti-gettered argon atmosphere. Each ingot was melted several times to obtain chemical homogeneity and finally cast into a water-cooled copper mold to obtain a rectangular plate with the dimension of $\sim 80 \times 10 \times 1 \mathrm{~mm}$. The thickness was further reduced to $0.4 \mathrm{~mm}$ by grinding and mechanical polishing for mechanical characterization.

Surface Mechanical Attrition Treatment (SMAT). As pioneered by Lu and $\mathrm{Lu}^{38}$, the SMAT method was originally developed to induce surface nanocrystallization in crystalline metals. Here, it was exploited to enhance the room-temperature plasticity of bulk metallic glasses (BMGs). Similar to classic shot peening, the SMAT method also utilizes the flying shots to impact randomly on the surface of a material, from which the surface plastic deformation are expected to alter the local material structure and residual stress profiles [Fig. 2(a)]. Nevertheless, compared to shot peening, the SMAT process also displays its distinctions in the following aspects: (a) a more stringent requirement for the roundness of shots to minimize the risk of surface wear damage, (b) larger shots, $\sim 300 \mu \mathrm{m}$ to $\sim 10 \mu \mathrm{m}$ in diameter, being used than those in the classic shot peening, which are $\sim 200 \mu \mathrm{m}$ to $\sim 1 \mathrm{~mm}$ in diameter, and (c) the randomness in the incident angle of the shots as compared to the nearly unidirectional impacts in the shot peening, which are usually close to 90 degrees.

In our SMAT set-up, a $20 \mathrm{kHz}$ ultrasonic transducer was employed as the impulse source to excite a number of hard spherical shots, which were placed in a reflecting chamber (including an ultrasonic concentrator), to impact on the sample surface. Because of the high frequency of the system, the entire surface of the treated component was peened with a very high number of impacts over a short period of time. Such an experimental set up enabled a much better control of energy transfer into the treated material than otherwise, which was already found crucial to the SMATinduced structural alteration in the treated material ${ }^{13}$.

Tensile tests and Digital Imaging Correlation (DIC). The tensile tests were performed at room temperature and at a strain rate of $10^{-4} \mathrm{~s}^{-1}$ in a universal tensile test machine MTS Alliance RT/50. The tensile strain was measured by the digital image correlation (DIC) method using the commercial package Vic-3 $\mathrm{D}^{\mathrm{TM}}$ (Correlated Solutions Inc., Columbia, SC, USA) (see Supplementary Information).

Hole-Drilling Method for Residual Stress Measurement. Residual stresses were measured using the incremental hole-drilling strain-gage method ${ }^{20}$. During the measurement, $2-\mathrm{mm}$ holes were drilled to release the locked-in residual stress inside the material, then the stress-release induced strain was measured at the surrounding area of the hole by a strain gage rosette (with 3 strain gage located at $0,135,270$ degrees direction), and finally the original residual stress was back out using finite element simulation.

X-Ray Diffraction (XRD). To check the atomic structure of the as-cast and SMATed $\mathrm{BMGs}$, the X-ray diffraction (XRD) tests were carried out with Co K $\alpha$ radiation using a Bruker AXS D8 Discover X-ray diffractometer.

Transmission Electron Microscopy (TEM). The atomic structure of the $\mathrm{Cu}-\mathrm{Zr}$ based BMG plates before and after SMAT were examined by transmission electron microscopy (TEM) on a JEOL $2010 \mathrm{~F}$ operated at $200 \mathrm{kV}$. Following the previous $\operatorname{method}^{39}$, the TEM specimens, taken at the different depths below the SMAT surface, were prepared sequentially by mechanical polishing, dimple-grinding and ionmilling.

Scanning Electronic Microscopy (SEM). The surface morphology and the fracture features of all the specimens after tensile failure were investigated by scanning electron microscopy on a Hitachi S4800 field emission gun (FEG) SEM.

Nanoindentation. Nanoindentation was carried out on the cross section of the BMG plates before and after SMAT on the Hysitron ${ }^{\mathrm{TM}}$ NanoIndenter TI950 using a Berkovich indenter. To avoid the surface pile-up effect, hardness was measured by direct imaging the indents.

Differential Scanning Calorimetry (DSC). The differential scanning calorimetry (DSC) experiments were performed on the as-cast and SMATed samples, using a Perkin Elmer Diamond DSC under high-purity flowing Ar at the heating rate of $20 \mathrm{~K} /$ min. Aluminum pans were used as sample holders. For all the DSC runs, two successive runs were recorded with the second run serving as a baseline. The calorimeter was calibrated for temperature and energy at the heating rate of $20 \mathrm{~K} / \mathrm{min}$ with high purity indium and zinc standards, giving an experimental accuracy for the temperature and the enthalpy of about $\pm 1 \mathrm{~K}$ and $\pm 0.5 \mathrm{~J} / \mathrm{g}$, respectively. For consistency, a 60- $\mu \mathrm{m}$ deep SMAT affected zone taken from the treated sample with a total mass $\sim 10 \mathrm{mg}$ was measured for all BMGs.

1. Bourhis, E. L. Glass: Mechanics and Technology. (Wiley-VCH Verlag GmbH \& Co. $\mathrm{KGaA}, 2008$ ). 
2. Kumar, G., Tang, H. X. \& Schroers, J. Nanomoulding with amorphous metals. Nature 457, 868-872 (2009).

3. Zberg, B., Uggowitzer, P. J. \& Loffler, J. F. MgZnCa glasses without clinically observable hydrogen evolution for biodegradable implants. Nat Mater 8, 887-891 (2009).

4. Ritchie, R. O. The conflicts between strength and toughness. Nat. Mater. 10, 817-822 (2011).

5. Hofmann, D. C. et al. Designing metallic glass matrix composites with high toughness and tensile ductility. Nature 451, 1085-1089 (2008).

6. Sarac, B. \& Schroers, J. Designing tensile ductility in metallic glasses. Nat. Commun. 4, 2158 (2013).

7. Jang, D. C. \& Greer, J. R. Transition from a strong-yet-brittle to a stronger-andductile state by size reduction of metallic glasses. Nat. Mater. 9, 215-219 (2010).

8. Schuh, C., Hufnagel, T. \& Ramamurty, U. Mechanical behavior of amorphous alloys. Act. Mater. 55, 4067-4109 (2007).

9. Zhang, Z. F., Eckert, J. \& Schultz, L. Difference in compressive and tensile fracture mechanisms of $\mathrm{Zr}_{59} \mathrm{Cu}_{20} \mathrm{Al}_{10} \mathrm{Ni}_{8} \mathrm{Ti}_{3}$ bulk metallic glass. Act. Mater. 51, 1167-1179 (2003).

10. Qu, R. T., Zhang, Q. S. \& Zhang, Z. F. Achieving macroscopic tensile plasticity of monolithic bulk metallic glass by surface treatment. Scripta Mater. 68, 845-848 (2013).

11. Wu, Y., Xiao, Y., Chen, G., Liu, C. T. \& Lu, Z. P. Bulk Metallic Glass Composites with Transformation-Mediated Work-Hardening and Ductility. Adv. Mater. 22, 2770-2773 (2010)

12. Schroers, J., Kumar, G., Hodges, T. M., Chan, S. \& Kyriakides, T. R. bulk metallic glasses for biomedical applications. JOM 61, 21-29 (2009).

13. Chan, H. L., Ruan, H. H., Chen, A. Y. \& Lu, J. Optimization of the strain rate to achieve exceptional mechanical properties of 304 stainless steel using high speed ultrasonic surface mechanical attrition treatment. Acta Mater. 58, 5086-5096 (2010)

14. Zhang, Y., Wang, W. H. \& Greer, A. L. Making metallic glasses plastic by control of residual stress. Nat. Mater. 5, 857-860 (2006).

15. Ramamurty, U., Jana, S., Kawamura, Y. \& Chattopadhyay, K. Hardness and plastic deformation in a bulk metallic glass. Acta Mater. 53, 705-717 (2005).

16. Wu, F. F., Zhang, Z. F. \& Mao, S. X. Size-dependent shear fracture and global tensile plasticity of metallic glasses. Acta Mater. 57, 257-266 (2009).

17. Schajer, G. S. Advances in Hole-Drilling Residual Stress Measurements. Exp. Mech. 50, 159-168 (2009).

18. Nikulari, A., Lu, J. \& Flavennot, J. F. Measurement of residual-stress distribution by the incremental hole-drilling method. Exp. Mech. 25, 175-185 (1985).

19. Lu, J. in Handbook on residual stress (Published by Experimental Mechanics, Inc. through the American Institute of Physics, 2005).

20. ASTM. Vol. ASTM Standard Test Method E837-08 (American Society for Testing and Materials, West Conshohocken, 2008).

21. Dmowski, W. et al. Structural rejuvenation in a bulk metallic glass induced by severe plastic deformation. Acta Mater. 58, 429-438 (2010).

22. Slipenyuk, A. \& Eckert, J. Correlation between enthalpy change and free volume reduction during structural relaxation of $\mathrm{Zr}_{55} \mathrm{Cu}_{30} \mathrm{Al}_{10} \mathrm{Ni}_{5}$ metallic glass. Scripta Mater. 50, 39-44 (2004).

23. Bei, H., Xie, S. \& George, E. Softening caused by profuse shear banding in a bulk metallic glass. Phys. Rev. Lett. 96, 105503 (2006).

24. Yang, Y., Ye, J. C., Lu, J., Liu, F. X. \& Liaw, P. K. Effects of specimen geometry and base material on the mechanical behavior of focused-ion-beam-fabricated metallic-glass micropillars. Acta Mater. 57, 1613-1623 (2009).

25. Wang, L., Bei, H., Gao, Y. F., Lu, Z. P. \& Nieh, T. G. Effect of residual stresses on the hardness of bulk metallic glasses. Acta Mater. 59, 2858-2864 (2011).

26. Guo, H. et al. Tensile ductility and necking of metallic glass. Nat. Mater. 6 , 735-739 (2007).

27. Cao, Q. P. et al. Effect of pre-existing shear bands on the tensile mechanical properties of a bulk metallic glass. Acta Mater. 58, 1276-1292 (2010).

28. Scudino, S., Jerliu, B., Surreddi, K. B., Kühn, U. \& Eckert, J. Effect of cold rolling on compressive and tensile mechanical properties of $\mathrm{Zr}_{52.5} \mathrm{Ti}_{5} \mathrm{Cu}_{18} \mathrm{Ni}_{14.5} \mathrm{Al}_{10}$ bulk metallic glass. J. Alloys and Comp. 509, S128-S130 (2011).
29. Cui, J. W., Calin, M., Eckert, J. \& Zhang, Z. F. Tensile fracture dynamics and intrinsic plasticity of metallic glasses. Appl. Phys. Lett. 102, 031908 (2013).

30. Ye, J. C., Lu, J., Liu, T. C., Wang, Q. \& Yang, Y. Atomistic free-volume zones and inelastic deformation of metallic glasses. Nat. Mater. 9, 619-623 (2010).

31. Yang, Y. et al. Fractal growth of the dense-packing phase in annealed metallic glass imaged by high-resolution atomic force microscopy. Acta Mater. 60, 5260-5272 (2012).

32. Liu, Y. H. et al. Characterization of Nanoscale Mechanical Heterogeneity in a Metallic Glass by Dynamic Force Microscopy. Phys. Rev. Lett. 106, 125504 (2011).

33. Fujita, T. et al. Low temperature uniform plastic deformation of metallic glasses during elastic iteration. Acta Mater. 60, 3741-3747 (2012).

34. Argon, A. S. Plastic deformation in metallic glasses. Acta Metall. 27, 47-58 (1979).

35. Dmowski, W., Iwashita, T., Chuang, C. P., Almer, J. \& Egami, T. Elastic Heterogeneity in Metallic Glasses. Phys. Rev. Lett. 105, 205502 (2010).

36. Egami, T. Mechanical failure and glass transition in metallic glasses. J. Alloys and Comp. 509, S82-S86 (2011)

37. Carlos, A. P. Review: Flow and fracture in amorphous alloys. J. Mater. Sci. 10, 1194-1227 (1975).

38. Lu, J. \& Lu, K. Surface nanocrystallization (SNC) of Materials and its effecton mechanical behavior. Comprehensive structural integrity 8, 496-528 (2003)

39. Tian, J. W., Shaw, L. L., Wang, Y. D., Yokoyama, Y. \& Liaw, P. K. A study on the surface severe plastic deformation behavior of a Zr-based bulk metallic glass (BMG). Intermetallics 17, 951-957 (2009).

\section{Acknowledgments}

Q.W., J.H. \& J.L. acknowledge the support from the National Basic Research Program of China (MOST 973 Program) under the grant 2012CB932203, Research Grants Council of the Hong Kong Special Administrative Region of China under grants GRF/CityU519110, and the Croucher Foundation under grant CityU9500006. The research of Y.Y. is supported by the City University of Hong Kong through the start-up grant for newly recruited academic staff, and the Research Grant Council (RGC), the Hong Kong Government, through the General Research Fund (GRF) with the grant number CityU530711. C.T.L. acknowledges the support by the Research Grant Council, the Hong Kong Government, through the General Research Fund under project CityU/522110. Q.W. would also like to thank Prof. Y.D.D. at Shanghai University for supportive discussions and acknowledge the financial supports provided by Natural Science Foundation of China (Grant No. 50871063, 51171099) and from the 085 Project in Shanghai University.

\section{Author Contributions}

J.L. designed the project. Q.W. and J.H. carried out the experiments. Q.W. and Y.Y. analyzed the data. J.L., Y.Y., C.T.L., Q.W. and H.H.R. contributed to the discussion of the results. Y.Y. and Q.W. wrote the manuscript.

\section{Additional information}

Supplementary information accompanies this paper at http://www.nature.com/ scientificreports

Competing financial interests: The authors declare no competing financial interests. How to cite this article: Wang, Q. et al. Superior Tensile Ductility in Bulk Metallic Glass with Gradient Amorphous Structure. Sci. Rep. 4, 4757; DOI:10.1038/srep04757 (2014).

This work is licensed under a Creative Commons Attribution-NonCommercialShareAlike 3.0 Unported License. The images in this article are included in the article's Creative Commons license, unless indicated otherwise in the image credit; if the image is not included under the Creative Commons license, users will need to obtain permission from the license holder in order to reproduce the image. To view a copy of this license, visit http://creativecommons.org/licenses/by-nc-sa/3.0/ 